\title{
Effects of Inclination and Magnetic Field on Natural Convection Flow Induced by a Vertical Temperature
}

\author{
M. N. Kherief ${ }^{1 \dagger}$, K. Talbi ${ }^{2}$ and F. Berrahil ${ }^{2}$ \\ ${ }^{1}$ Department of Mechanics Faculty of Science of Engineer, University of the 20 -08-55 Skikda. Algiers \\ ${ }^{2}$ University Mentouri-Constantine Faculty - Engineerings Mechanical Department of Engineering. Algiers \\ †Corresponding Author Email: Kherief2006@yahoo.fr
}

(Received February 23, 2010; accepted August 3, 2010)

\begin{abstract}
A numerical program is been developed to simulate the natural convection in a rectangular cavity in presence of a magnetic field. The cavity in filled with mercury with a Prandtl number equal to 0.024 . The flow is induced by a vertical temperature gradient. This type of configuration concerns the crystal growth using the Bridgman vertical method. The mass, momentum and energy equations, adopting the Boussinesq approximation, are solved numerically using the finite-volume method in conjunction with the SIMPLER algorithm the flow under consideration is steady, laminar and two-dimensional. The temperature gradients are assumed to be weak. The results show that the dynamic and temperature fields are strongly affected by variations of the magnetic field intensity and the angle of inclination. Numerical simulations have been carried out considering different combinations of Grashof and Hartmann numbers to study their effects on the streamlines, the isotherms and the Nusselt number.
\end{abstract}

Keywords: Thermal convection, rectangular enclosure, magnetic field, finite-volume, Lorentz force.

\section{NOMENCLATURE}

$\begin{array}{ll}A & \text { Aspect ratio } \\ B & \text { Vector of the magnetic field T } \\ b & \text { Source term } \\ C & \text { Cold } \\ F & \text { Electromagnetic force } N / m^{3} \\ G r & \text { Grashof number } \\ g & \text { Acceleration of gravity } m / s^{2} \\ H & \text { Heat } \\ H & \text { Width of the cavity } m \\ \mathrm{Ha} & \text { Hartmann number } \\ \mathrm{J} & \text { Vector of density of the electrical current } A / \mathrm{m}^{2} \\ \mathrm{~L} & \text { Length of the cavity } m \\ \overline{N u} \mid & \text { Average Nusselt number } \\ \mathrm{P} & \text { Pressure } N / \mathrm{m}^{2} \\ \mathrm{Pr} & \text { Prandtl number } \\ \mathrm{T} & \text { temperature } K \\ \mathrm{t} & \text { Dimensionless time }\end{array}$

\section{INTRODUCTION}

Natural convection in closed enclosures has been extensively studied numerically and experimentally. The study of thermal convection in inclined enclosures is motivated by a desire to find out what effect slope would have on certain thermally driven flows which are found in many engineering applications. These applications include: building systems containing multilayered walls, double windows, and air gaps in u Horizontal velocity $\mathrm{m} / \mathrm{s}$

U Dimensionless horizontal velocity

v Vertical velocity $\mathrm{m} / \mathrm{s}$

V Dimensionless vertical velocity

$\mathrm{X}$ Cartesian coordinate in the horizontal direction

$\mathrm{Y}$ Cartesian co-ordinate in the vertical direction

$\alpha \quad$ Thermal diffusivity $m^{2} / s$

$\beta_{t} \quad$ Thermal coefficient of expansion $1 / K$

$\Delta t \quad$ Dimensionless increment of time

$\varphi \quad$ Electric potential $V$

$\Phi$ Generalized function

$\rho$ Mass density $\mathrm{kg} / \mathrm{m}^{3}$

$\gamma \quad$ Angle of inclination

$\theta$ Dimensionless temperature

$\sigma \quad$ Electric conductivity $1 / \Omega . m$

$v$ Kinematic viscosity $\mathrm{m}^{2} / \mathrm{s}$

unventilated spaces; energy systems such as solar collectors, storage devices, furnaces, heat exchangers, and nuclear reactors; material processing equipment such as melting and crystal growth reactors. Thermally driven flows are also found in large scale geophysical, astrophysical, and environmental phenomena. Most of the research work that has been carried out in this area was focused on enclosures that were differentially heated in one direction (vertically or horizontally) with 
adiabatic side walls in the other direction. Rather little work has been carried out considering more complex thermal boundary conditions that are normally found in most of the aforementioned practical applications. In these applications, the imposed temperature gradient is neither horizontal nor vertical.

Thermal convection in enclosures heated from below and cooled from above is of special interest because of flow mode transition that had been observed and reported by many researchers (Hart 1971; Corcione 2003). A numerical investigation on the effect of various thermal conditions of cavity side walls on flowmode transition of natural convection in rectangular horizontal enclosures, heated from below and cooled on the top. Different width-height aspect ratios were considered. Rayleigh numbers, based on cavity height, were betweens $10^{3}$ to $10^{6}$. Corcione results have shown that bidirectional differential heating has a significant effect on flow mode-transition of natural convection inside horizontal cavities.

Some of these bidirectional differential heating configurations considered in involved temperature discontinuities at the cavity corners. No discussion was provided on the effect of these temperature discontinuities on the calculated Nusselt numbers. numerically investigated the effect of angle of inclination on flow mode-transition in an inclined rectangular enclosure heated from below and cooled from above with two insulated side walls (Song 1996). Different aspect ratios were considered and Rayleigh number ranged from $1.5 \times 10^{3}$ to $2 \times 10^{4}$. Flow modetransition and Hysteresis phenomenon for $\mathrm{Ra}>2000$ have been demonstrated.

When the fluid is electrically conducting and exposed to a magnetic field the Lorentz force is also active and interacts with the buoyancy force in governing the flow and temperature fields. Employment of an external magnetic field has increasing applications in material manufacturing industry as a control mechanism since the Lorentz force suppresses the convection currents by reducing the velocities. Study and thorough understanding of the momentum and heat transfer in such a process is important for the better control and quality of the manufactured products.

The study of Oreper (1983) shows that the magnetic field suppresses the natural-convection currents and the magnetic field strength is one of the most important factors for crystal formation. Ozoe and Maruo (1987) numerically investigated the natural convection of a low Prandtl number fluid in the presence of a magnetic field and obtained correlations for the Nusselt number in terms of Rayleigh, Prandtl and Hartmann numbers. Garandet (1992) proposed an analytical solution to the governing equations of magnetohydrodynamics to be used to model the effect of a transverse magnetic field on natural convection in a two-dimensional cavity.

Seth and Ghosh (1986) proposed unsteady hydromagnetic flow in a rotating channel in the presence of inclined magnetic field. Ghosh (1991) proposed a note on steady and unsteady hydromagnetic flow in a rotating channel in the presence of inclined magnetic field. Ghosh (2001) proposed a note on unsteady hydromagnetic flow in a rotating channel permeated by an inclined magnetic field in the presence of an oscillator. Ghosh and Pop (2002) proposed a note on a hydromagnetic flow in a slowly rotating system in the presence of an inclined magnetic field. Ghosh et al. (2010) proposed transient hydromagnetic flow in a rotating channel permeated by an inclined magnetic field with magnetic induction and Maxwell displacement current effects.

Rudraiah (1995) numerically investigated the effect of a transverse magnetic field on natural-convection flow inside a rectangular enclosure with isothermal vertical walls and adiabatic horizontal walls and found out that a circulating flow is formed with a relatively weak magnetic field and that the convection is suppressed and the rate of convective heat transfer is decreased when the magnetic field strength increases. Alchaar (1995) numerically investigated the natural convection in a shallow cavity heated from below in the presence of an inclined magnetic field and showed that the convection modes inside the cavity strongly depend on both the strength and orientation of the magnetic field and that horizontally applied magnetic field is the most effective in suppressing the convection currents.

Al-Najem (1998) used the power law control volume approach to determine the flow and temperature fields under a transverse magnetic field in a tilted square enclosure with isothermal vertical walls and adiabatic horizontal walls at Prandtl number of 0.71 and showed that the suppression effect of the magnetic field on convection currents and heat transfer is more significant for low inclination angles and high Grashof numbers. The present study considers laminar natural convection flows in the presence of a magnetic field in an inclined rectangular enclosure heated from isothermal vertical walls and adiabatic horizontal walls. The object of the study is to obtain numerical solutions for the velocity and temperature fields inside the enclosure and to determine the effects of the magnetic field strength and direction, the aspect ratio and the inclination of the enclosure on the transport phenomena.

\section{GEOMETRY AND MATHEMATICAL MODEL}

The geometry considered is a rectangular enclosure having a length $\mathrm{L}$ and a width $\mathrm{H}$, thus with an aspect ratio $\mathrm{A}=\mathrm{L} / \mathrm{H}=4$, filled completely with a molten metal, the Prandtl number of which is $\operatorname{Pr}=0.024$. The horizontal walls of the enclosure are maintained at different temperatures, the bottom wall at $\mathrm{T}_{H}$ and the top wall at $\mathrm{T}_{\mathrm{C}},\left(\mathrm{T}_{\mathrm{H}}>\mathrm{T}_{\mathrm{C}}\right)$. The other walls are supposed to be adiabatic. The inclination of the cavity was also considered, with a varying angle $\gamma$ between the heated wall and the horizontal line. The flow is subjected to the action of an external uniform and constant magnetic field. MHD flow, likely to develop in this enclosure, is governed by the equations of continuity, momentum, energy conservation, the Ohm's law and the conservation the electrical potential. The geometrical configuration is described in the Fig. 1. 


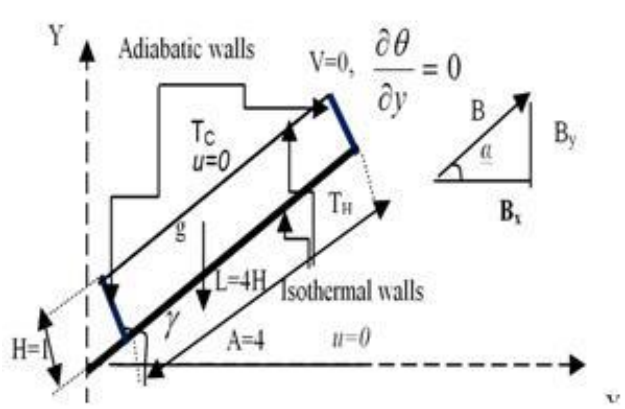

Fig. 1. Geometry of the problem.

We will adopt the following simplifying assumptions: the flow is laminar, the physical properties of the fluid are supposed constant, the viscous dissipation and the Joule effect are negligible, the approximation of Boussinesq is valid and the induced magnetic field is negligible (on the scale of the laboratory) because the magnetic Reynolds number $\mathrm{R}_{\mathrm{M}}<<1$, (Moreau 1991). As a result, the equations of MHD are written as follows:

$j=\sigma(-\nabla \phi+V \times B)$

$\nabla \cdot \vec{j}=0$

$\vec{F}_{E M}=\vec{j} \times \vec{B}$

$\vec{j}=\sigma[\vec{V} \times \vec{B}]$

$\vec{F}_{E M}=\sigma[\vec{V} \times \vec{B}] \times \vec{B}$

(5)

This gives:

$F_{E M}=\sigma \cdot B_{0}^{2}\left[\left(v \cos \alpha \sin \alpha-u \sin ^{2} \alpha\right) e_{x}+\left(u \sin \alpha \cos \alpha-v \cos ^{2} \alpha\right) e_{y}\right]$

The dimensionless equations describing the flow are as follows.

At $t=0: U=V=0$.

At $t>0$

$\frac{\partial U}{\partial X}+\frac{\partial V}{\partial Y}=0$

$\frac{\partial U}{\partial t}+U \frac{\partial U}{\partial X}+V \frac{\partial U}{\partial Y}=\operatorname{Gr} \theta \sin \gamma-\frac{\partial P}{\partial X}+\left[\frac{\partial^{2} U}{\partial X^{2}}+\frac{\partial^{2} U}{\partial Y^{2}}\right]+F_{\text {EUX }}$

$\frac{\partial V}{\partial t}+U \frac{\partial V}{\partial X}+V \frac{\partial V}{\partial Y}=\operatorname{Gr} \theta \cos \gamma-\frac{\partial P}{\partial Y}+\left[\frac{\partial^{2} V}{\partial X^{2}}+\frac{\partial^{2} V}{\partial Y^{2}}\right]+F_{E U Y}$

$\frac{\partial \theta}{\partial t}+U \frac{\partial \theta}{\partial X}+V \frac{\partial \theta}{\partial Y}=\frac{1}{\operatorname{Pr}}\left[\frac{\partial^{2} \theta}{\partial X^{2}}+\frac{\partial^{2} \theta}{\partial Y^{2}}\right]$

We note that $\mathrm{p}$ is the dimensionless pressure, $\mathrm{U}$ and $\mathrm{V}$ are the dimensionless velocity components, $\theta$ is the dimensionless temperature, $\rho$ it is supposed linear distribution. $\alpha, \mathrm{g}, \beta$ and $\sigma$ are respectively the thermal diffusivity, the gravity acceleration, the thermal coefficient of expansion and the electrical conductivity. The dependant variables are nondimensionnalized using the following characteristic parameters: $\mathrm{H}, \mathrm{H}^{2} / v, v / \mathrm{H}, \rho$ $(v / \mathrm{H})^{2}$ and $\mathrm{T}_{\mathrm{H}}-\mathrm{T}_{\mathrm{C}}$ for the length, time, velocity, the pressure and the temperature respectively. $v, \rho$ and $t$ represent the kinematic viscosity of the molten metal, the density and time respectively.

The electrical potential $\varphi$ equation is obtained by taking the divergence of the Ohm's law (Series 1991). The dimensionless components of the Lorentz force $\mathrm{F}$ in the $\mathrm{x}$ and $\mathrm{y}$ directions appear in the $\mathrm{x}$ and $\mathrm{y}$ momentum equations respectively. In addition the boundaries are electrically insulated; therefore the electric potential $\varphi$ is constant. The MHD control parameters of flow are: $G r=g \beta\left(T_{H}-T_{C}\right) H^{3} / v^{2}, H a=B_{0} H \sqrt{\sigma / \rho v}$ and $\quad \operatorname{Pr}=v / \alpha$ indicating the Grashof number, the Hartmann number and the Prandtl number respectively.

\subsection{Boundary Conditions}

The following boundary conditions are incorporated:

- At $X=0$ and $X=A, U=V=d \theta / d Y=0$.

- At $Y=0, U=V=0, \theta=1$ and at $Y=1, \theta=0$.

\section{NUMERICAL METHOD}

The finite-volume method is used for the numerical resolution the system of transport Eq. (11).

$\frac{\partial \varphi}{\partial t}+\frac{\partial\left(U_{i} \varphi\right)}{\partial X_{i}}=\frac{\partial}{\partial X_{i}}\left(\Gamma \varphi \frac{\partial \varphi}{\partial X_{i}}\right)+S_{\varphi}$

The discretized form is:

$A_{P} \varphi_{P}=A_{E} \varphi_{E}+A_{W} \varphi_{W}+A_{N} \varphi_{N}+A_{S} \varphi_{S}+b$

The Eq. (12) are solved using the SIMPLER algorithm (Patankar 1980) the temporal derivative is discretized using the implicit scheme. Concerning the spatial discretization, all the convective and the diffusive terms are discretized using the central differencing scheme.

In this study, we used a uniform grid of $112 \times 42$ in the $\mathrm{X}$ and $\mathrm{Y}$ directions respectively. The time step $\Delta \mathrm{t}=10^{-}$ ${ }^{4}$. At this time one expects that the assessment of mass conservation and energy is satisfied. Convergence is attained when the difference between the average Nusselt numbers of the cold wall and the hot wall is negligible and the difference between the velocities and temperatures evaluated at times $t$ and $t+\Delta t$ is negligible. The average Nusselt number is defined in the present study by:

$$
\overline{\mid N u}_{Y=0,1}=\int_{0}^{A} \frac{\partial \theta}{\partial Y} d X .
$$

\section{Results ANd Discussions}

The computer program was validated initially with the solution Benchmark (Davis 1983) of the natural convection without the presence of the magnetic field. After that, we confronted our results with the results obtained by BenHadid (1997) and Gelfgat (2001) where the magnetic field is applied. In a third case, both a magnetic field and a slope of the enclosure are considered and the results compared with those of Mehmet (2006). 


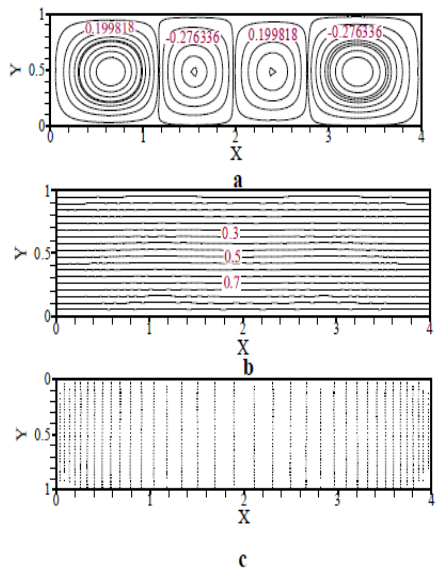

1. $\mathrm{Gr}=10^{5}$
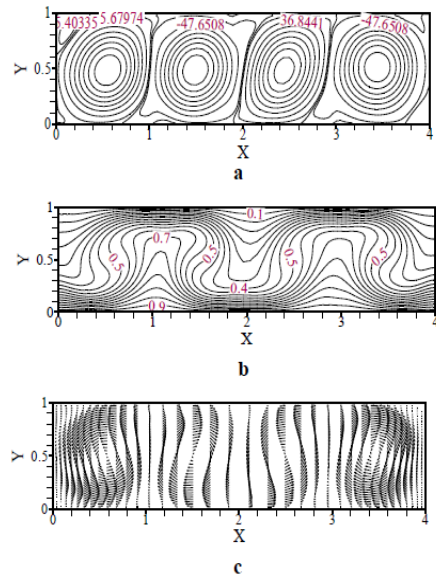

2. $\mathrm{Gr}=5.10^{5}$
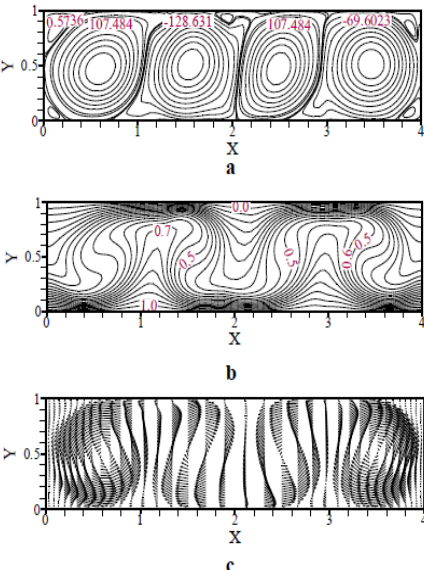

3. $\mathrm{Gr}=10^{6}$

Fig. 2. Structure of the flow represented by: a) contours of stream function, b) isothermal c) velocity vectors for: $\operatorname{Pr}=0.024, \mathrm{Ha}=0, \mathrm{~A}=4, \alpha=0, \gamma=0$ and for various Grashof number $\mathrm{Gr}$.

\subsection{Influence of the Grashof Number}

In effect, results obtained show that the flow and the thermal fields illustrated in the Figs. 2.1a and 2.1b, Figs. 2.2a and 2.2b and Figs. 2.3a; and 2.3b, respectively relatively intense straining of the fluid for large values of $\mathrm{Gr}$. We note on these figures the evolution of the stream function by increasing the $\mathrm{Gr}$ number. At the beginning, circulation is stable and gives four cells distributed on the whole of the field, Fig. 2.1a. Then, one notices light modifications which appear by increasing the number of Gr. For $\mathrm{Gr}=10^{6}$, small recirculation zones, also called "vortices", were observed between the principal cell in the middle of the upper wall and in the top-right and the bottom-left corners, Fig. 2.3a.

The thermal field of the flow is presented in Figs. 2.1b, $2.2 \mathrm{~b}$ and $2.3 \mathrm{~b}$. It should be noted that for a weak $\mathrm{Gr}$ number, no good stratification of the isotherms with the horizontal walls of the enclosure is observed. Indeed when the Gr number increases, we notice the presence of the significant variations in temperature. This is explained by the existence of a convective transport dominating the flow (the acceleration of the particles cause this phenomenon). Figure 5 shows the average Nusselt number of the top wall, for Gr equal to $10^{5}$, $3.10^{5}$ and $10^{6}$. The convection flow along the two adiabatic vertical walls the beginning of the development of a multi-cell flow within the cavity, which enhanced effectiveness of heat transfer through top wall. This effect has been observed until $\mathrm{Gr}=10^{6}$, where the cross temperature gradient and the resulting multi-cell flow have become strong enough for heat transfer to be enhanced from the bottom wall to the top wall. In Fig. 2.1c, Fig. 2.2c and Fig. 2.3c, we present the distribution of the velocity vectors. We notice, whereas for a small number of Gr, the flow generates very weak velocity gradients, when the Gr number increases, the flow induced by the increasing buoyancy forces becomes animated. Significant velocity gradients are then localized near the walls, resulting in the production of vortices. This is well illustrated in Figs. 3 and 4.

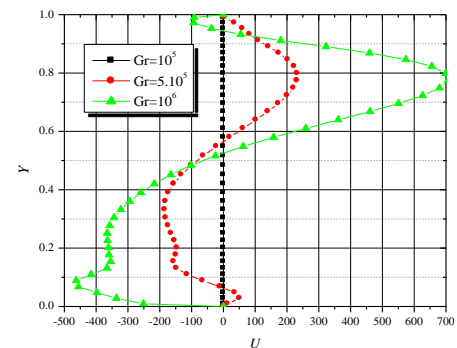

Fig. 3. Profiles of horizontal velocity component at $\mathrm{x}=2 ; \operatorname{Pr}=0.024, \mathrm{Ha}=0, \mathrm{~A}=4, \alpha=0, \gamma=0$.

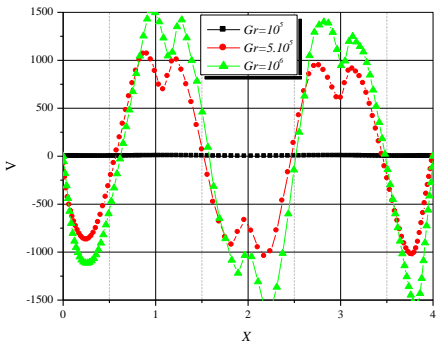

Fig. 4. Profiles of vertical velocity component at $\mathrm{Y}=0.5: \mathrm{Pr}=0.024, \mathrm{Ha}=0, \mathrm{~A}=4, \alpha=0, \gamma=0$.

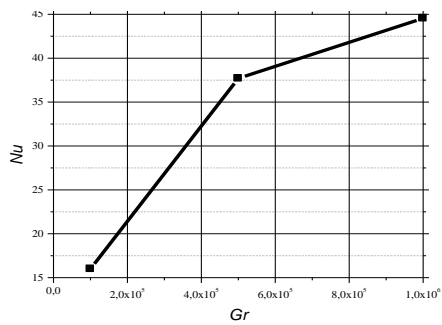

Fig. 5. Variation the average Nusselt number according to Grashof number $\mathrm{Gr}$ for: $\mathrm{Pr}=0.024, \mathrm{Ha}=0, \mathrm{~A}=4, \alpha=$ $0, \gamma=0$ and $\operatorname{Gr}=\left(10^{5}, 5.10^{5}\right.$ and $\left.10^{6}\right)$.

\subsection{Effects of the Hartmann Number}

Figure 6 and Fig. 10 show the effect of the magnetic field in the two directions $\mathrm{X}$ and $\mathrm{Y}$ using different values of the Hartmann number $(\mathrm{Ha}=0,10,50,100)$. 


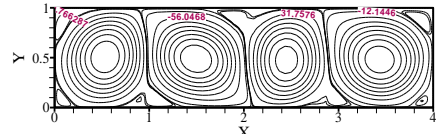

(a)

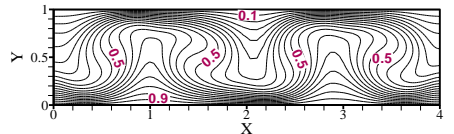

(b)

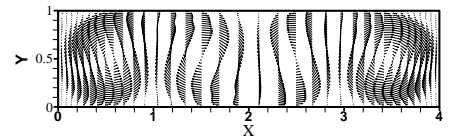

(c)

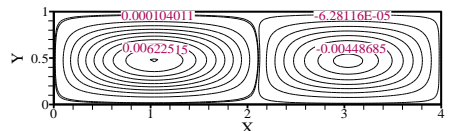

(a)

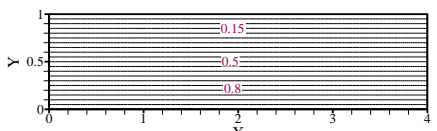

(b)

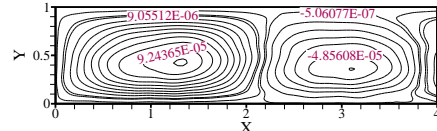

(a)

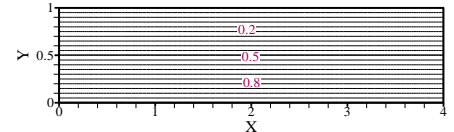

(b)

1. $\mathrm{Ha}=10$

2. $\mathrm{Ha}=50$

3. $\mathrm{Ha}=100$

Fig. 6. Structure of the flow represented by: a) contours of stream function, b) isothermal and c) velocity vectors for: $\mathrm{Gr}=10^{6}, \mathrm{Pr}=0.024, \mathrm{~A}=4, \alpha=0, \gamma=0$ and for various Hartmann number Ha.

To analyze the thermal natural convection was analyzed in the geometry for the following characteristic dimensionless numbers: $\mathrm{Gr}=10^{6}, \mathrm{Pr}=0.024, \mathrm{~A}=4$. We notice the results of the dynamic field presented, in the form of contours of the stream function, Fig. 6.1a and Fig. 10.1a. The figures show that for $\mathrm{Ha}=10$, the flow is characterized by four cells. When the intensity of the magnetic field in the $\mathrm{X}$ direction increases, the number of cells reduces to two cells. On the other hand, in the Y direction, with the same intensities, the flow structure changes.

For $\mathrm{Ha}=10$, the cells begin to lose their organized shape. When Ha is increased further, the flow becomes unstable and a somewhat perturbed cell is apparent. The effect of the magnetic field is significant when $\alpha$ takes the value of $90^{\circ}$. It is also noted that when the magnetic field is applied in the $\mathrm{Y}$ direction, the natural convection is clearly weakened. An elimination of the thermal stratification is obtained by the increase in the $\mathrm{Gr}$ number. However, an increase in the intensity of the magnetic field leads to isotherms nearly parallel to the horizontal walls explaining the elimination of the convective phenomena and the presence of the conductive phenomena. This also explains the deceleration of the thermal transfer as confirmed by the average Nusselt number Fig. 9 to Fig. 13. Concerning the horizontal and vertical normalized velocity profiles, they are shown in Figs7 and 8 (in the case of the $\mathrm{X}$ direction magnetic field) and Fig. 11 and Fig.12 (in the case of the Y-direction magnetic field) respectively.

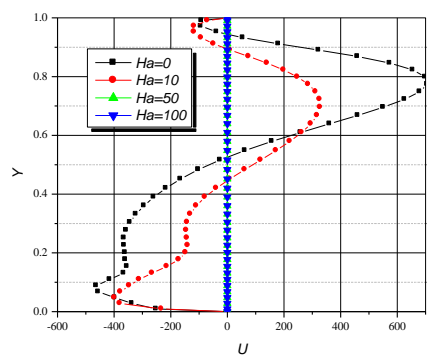

Fig.7. Profiles of horizontal velocity component at $\mathrm{x}=2$ : $\operatorname{Pr}=0.024, \mathrm{Gr}=10^{6}, \mathrm{~A}=4, \alpha=0, \gamma=0$.
It is clear from the results that as $\mathrm{Ha}$ are increased; the velocity components tend to diminish. In fact, for $\mathrm{Ha}=100$, their values are practically equal to zero in the major part of the cavity except near the end walls. It is thus clear that the use of a magnetic field can strongly decrease the flow intensity, but cannot completely inhibit fluid motion.

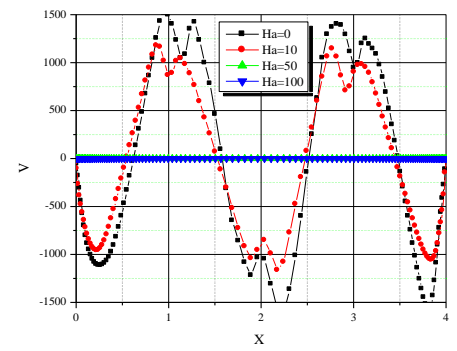

Fig. 8. Profiles of vertical velocity component at $\mathrm{Y}=0.5: \operatorname{Pr}=0.024, \mathrm{Gr}=10^{6}, \mathrm{~A}=4, \alpha=0, \gamma=0$.

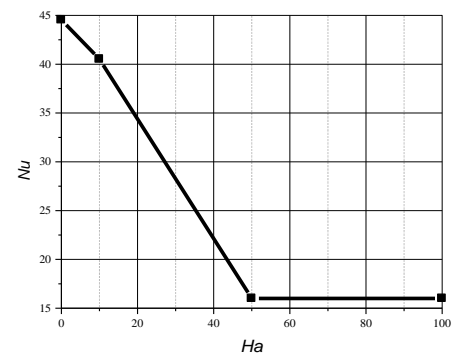

Fig.9. Variation the average Nusselt number according to $\mathrm{Ha}$ for: $\mathrm{Pr}=0.024, \mathrm{Gr}=106, \mathrm{~A}=4, \alpha=0^{0} . \gamma=0^{0}$.

\subsection{Influence Angle of Inclination $\gamma$}

The analysis of the Fig. 14.1, Fig. 14.2 and Fig. 14.3 shows that the enclosure slope has a strong effect on the flow and the heat transfer behavior. A single cell is obtained which appears to be completely stable, symmetrical and fills all the enclosure. On the other hand an accelerated flow with convective heat transfer along the vertical walls induced by the gravity forces is observed when the differently heated walls become vertical, Fig. 15 and Fig. 16. 


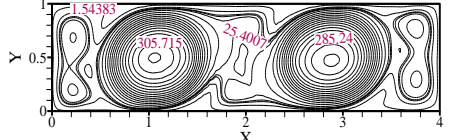

(a)

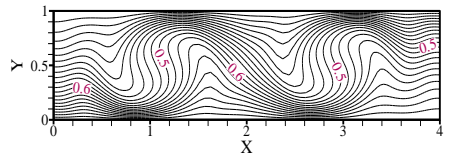

(b)

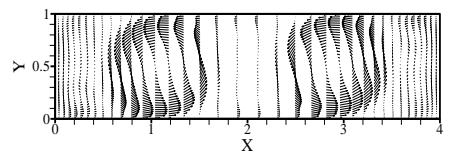

(c)

1. $\mathrm{Ha}=10$

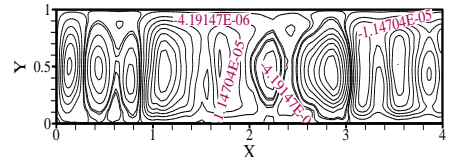

(a)

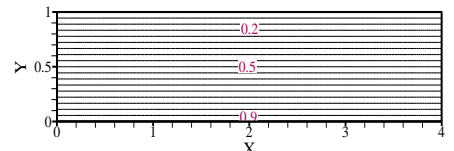

(b)

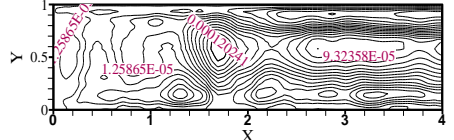

(a)

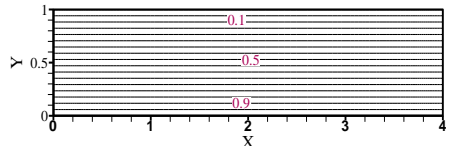

(b)

2. $\mathrm{Ha}=50$

3. $\mathrm{Ha}=100$

Fig. 10. Structure of the flow represented by: a) contours of stream function, b) isothermal and c) velocity vectors for: $\mathrm{Gr}=10^{6}, \mathrm{Pr}=0.024, \mathrm{~A}=4, \alpha=90^{\circ}, \gamma=0$ and for various Hartmann number.

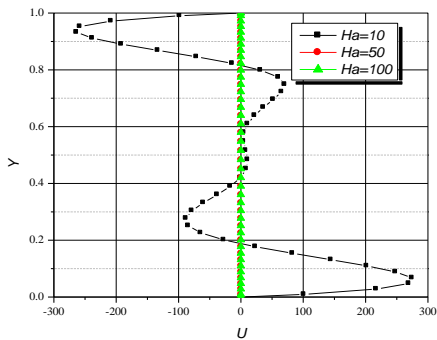

Fig.11. Profiles of hori-zontal velocity component at $\mathrm{x}=2: \operatorname{Pr}=0.024, \mathrm{Gr}=10^{6}, \mathrm{~A}=4, \alpha=90^{\circ}, \gamma=0$.

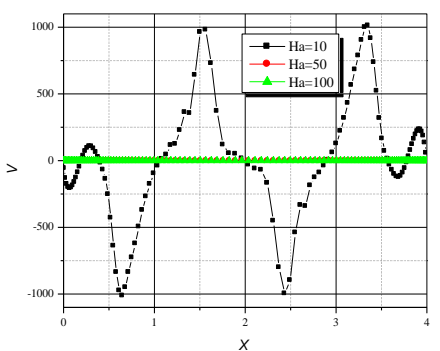

Fig.12. Profiles of vertical velocity component at $\mathrm{Y}=0.5: \operatorname{Pr}=0.024, \mathrm{Gr}=10^{6}, \mathrm{~A}=4, \alpha=90^{0}, \gamma=0$.

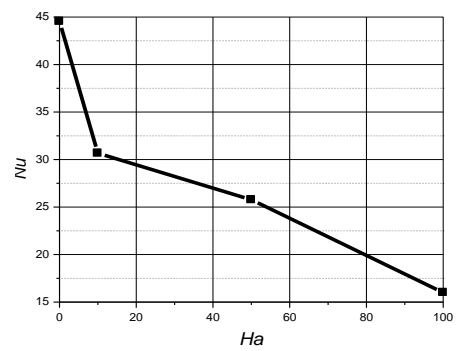

Fig. 13. Variation the average Nusselt number according to $\mathrm{Ha}$ for: $\mathrm{Pr}=0.024, \mathrm{Gr}=10^{6}, \mathrm{~A}=4, \alpha=90^{\circ}$, $\gamma=0$.

\section{CONCLUSION}

The results of the numerical simulation, enabled us to come out with several observations on the effects on the hydrodynamic and thermal structure of the flow, caused by temperature gradient. We summarize in what follows the principal results obtained:

Circulation and convection become stronger with increasing Grashof numbers but they are significantly suppressed by the presence of a strong magnetic field. Formation of multiple cells as a result of counterclockwise inclination greatly influences the temperature field. The average Nusselt number increases considerably with Grashof number since the circulation becomes stronger. The magnetic field significantly reduces the average Nusselt number by suppressing the convection streams.

\section{ACKNOWLEDGEMENTS}

Hot acknowledge of this work by the assistance provided by Laboratory for energetic CONSTANTINE.

\section{REFERENCES}

Alchaar, S., P. Vasseur, and E. Bilgen (1995). The effect of a magnetic field on natural convection in a shallow cavity heated from below. Chem. Eng. Commun. 134, 195-209.

Al-Najem, N.M., K.M. Khanafer, and M.M. El-Refaee (1998). Numerical study of laminar natural convection in tilted enclosure with transverse magnetic field. Int. J. Numer. Methods Heat Fluid Flow 8, 651-672.

BenHadid, H., and D. Henry (1997). Numerical study of convection in the horizontal Bridgman configuration under the action of a constant magnetic field. Part2. Three-dimensional flow. $J$. Fluid Mech. 333, 57- 83.

Corcione, M. (2003). Effects of the thermal boundary conditions at the sidewalls upon natural convection in rectangular enclosures heated from below and cooled from above. Int. J. Thermal Sci. 42, 199208. 


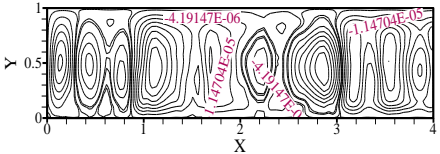

(a)

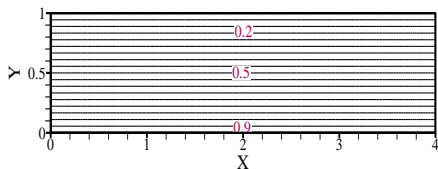

(b)

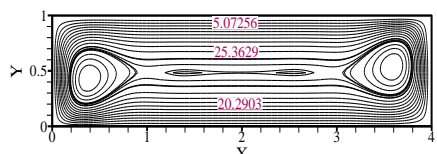

(a)

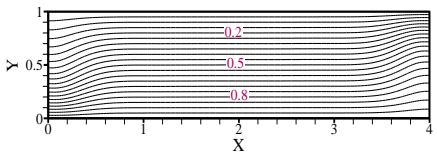

(b)

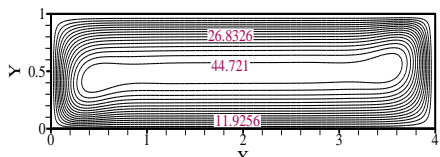

(a)

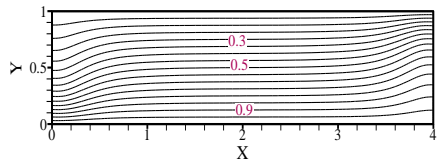

(b)

1. $\gamma=0^{\circ}$

2. $\gamma=45^{\circ}$

Fig. 14. Structure of the flow represented by: a) contours of stream function, $b$ ) the isotherm for: $\mathrm{Gr}=10^{6}, \operatorname{Pr}=0.024$, $\mathrm{A}=4, \alpha=90^{\circ}, \mathrm{Ha}=50$ and for various inclination values angle $\gamma$.

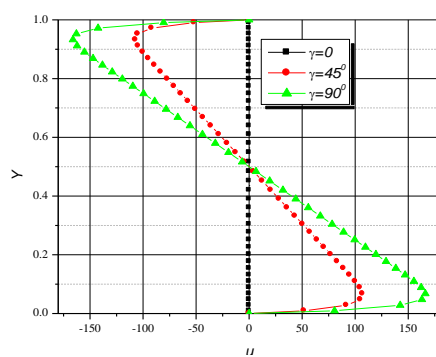

Fig. 15. Profiles of hori-zontal velocity component at $\mathrm{x}=2: \mathrm{Pr}=0.024, \mathrm{Gr}=106, \mathrm{Ha}=50, \mathrm{~A}=4, \alpha=90^{\circ}$.

Davis, G. and J.V.DE. Jones (1983). Natural convection in square cavity: comparison exercise. International Journal for Numerical Methods in fluids 3, 227-248.

Garandet, J.P., T. Alboussiere, and R. Moreau (1992). Buoyancy drive convection in a rectangular enclosure with a transverse magnetic field. Int $J$. Heat Mass Transfer 35, 741-748.

Gelfgat, A.Yu, P.Z. BarYoseph, and A. Solan (2001). Effect of axial magnetic field on three-dimensional instability of natural convection in a vertical Bridgman configuration. J. of Crystal Growth 230, 63-72.

Ghosh, S.K., O.A. Beg, J. Zueco, and V.R. Prasad (2010). Transient hydromagnetic flow in a rotatingchannel permeated by an inclined magnetic field with magnetic induction and Maxwell displacement current effects. Zeitschrift fur angewandte Mathematik und Physik ZAMP 61(1), 147-169.

Ghosh, S.K., and I. Pop (2002). A note on a hydromagnetic flow in a slowly rotating system in the presence of an inclined magnetic field. Magnetohydrodynamics 38(4), 377-384.

Ghosh, S.K. (2001). A note on unsteady hydromagnetic flow in a rotating channel permeated by an inclined magnetic field in the presence of an oscillator (Corrigendum and Addendum). Czechoslovak Journal of Physics 51(8), $799-804$.

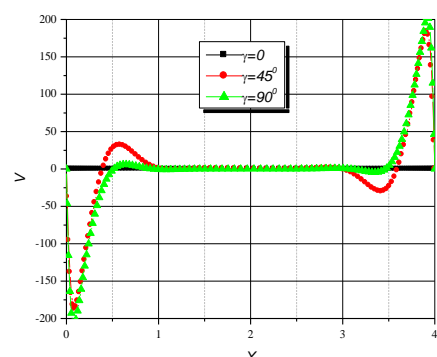

Fig.16. Profiles of verti-cal velocity component at $\mathrm{Y}=0.5 ; \mathrm{Pr}=0.024, \mathrm{Gr}=106, \mathrm{Ha}=50, \mathrm{~A}=4, \alpha=90^{\circ}$

Ghosh, S.K. (1991). A Note on steady and unsteady hydromagnetic flow in a rotating channel in the presence of inclined magnetic field. Int. J. Engng Sci. 29(8), 1013-1016.

Mehmet, C.E., and B. Elif (2006). Natural-convection flow under a magnetic field in an inclined rectangular enclosure heated and cooled on adjacent walls. Fluid Dynamics Research 38, 564-590.

Moreau, R. (1991). Magnetohydrodynamics. Kluwer Academic Publishers, 36-39.

Mukutmoni, D., and K.T. Yang (1994). Flow transitions and pattern selection of the RayleighBenard problem in rectangular enclosures. Sadhana - Academy Proceedings in Engineering Sciences 19 (5), 649-670.

Oreper, G.M., and J. Szekely (1983). The effect of an externally imposed magnetic field on buoyancy driven flow in a rectangular cavity. J. Cryst. Growth 64, 505-515.

Ozoe, H., and M. (1987). Maruo Magnetic and gravitational natural convection of melted silicontwo dimensional numerical computations for the rate of heat transfer. 198. JSME 30, 774-784.

Patankar, S.V. (1980). Numerical Heat Transfer and Fluid Flow. Hemisphere, Washington, DC. 
M.N. Kherief et al. / JAFM, Vol. 5, No.1, pp. 113-120, 2012.

Rudraiah, N., R.M. Barron, M. Venkatachalappa, and C.K. Subbaraya (1995). Effect of a magnetic field on free convection in a rectangular enclosure. Int. J. Eng. Sci. 33, 1075-1084.

Series, R., and D. Hurle (1991). The use of magnetic fields in semiconductor crystal growth. J. Cryst. Growth 113, 305-328.

Seth, G.S., and S.K. Ghosh (1986). Unsteady hydromagnetic flow in a rotating channel in the presence of inclined magnetic field. Int. J. Engng. Sci. 24(7), 1183-1193.

Song, C.Y., and P.Y. Tzeng (1996). Numerical study on mode-transition of natural convection in differentially heated inclined enclosures. Int. J. Heat Mass Transfer 39 (14), 2869-2882. 